\title{
Design of a Preamplifier Card for the Photomultiplier
}

\section{Tubes of a Gamma Camera}

\author{
Jorge Luis Domínguez Martínez \\ The Department of Nuclear Medicine, Surgical Clinical Hospital “Hermanos Ameijeiras”, Havana 10300, Cuba
}

\begin{abstract}
The service provided by the GC (Gamma Cameras) in the Nuclear Medicine departments fails because of their breakdown, generally due to the associated electronics and not to the physical detection components. For this reason, it was decided to develop an electronic system that allows the recovery and optimization of disused GC, starting with the design of the preamplifier for a PMT (photomultiplier tube). The circuit was designed and simulated and the list of components necessary for the construction of the preamplifier was generated, as well as the printed circuit board was designed for its assembly. By simulating the preamplifier this worked in linear mode. This determines that the amplitude of the output signal is proportional to the amount of charge delivered by the detector. This card allows an automatic adjustment of the signals of the PMT as do the modern GC.
\end{abstract}

Key words: Gamma camera, nuclear medicine, photomultiplier tube, preamplifier, Cuba.

\section{Introduction}

NM (nuclear medicine) is a therapeutic medical specialty or diagnostic imaging of functional-molecular type that uses unsealed sources; different types of compounds marked with radioactivity (radionuclide) are administered according to the organ or type of metabolism that you wish to evaluate following a specific physiological pathway, of a short half-life (only hours or a few days) and of low relative energy (which gives safety in its use, since the radiation absorbed in the patient is low); they are usually injected intravenously, inhaled, or taken orally [1-4]. In general, NM scans are noninvasive and have no adverse effects, so, for different pathologies, it has become the study of choice for clinical decision making [4]. When the radionuclide decays it emits gamma rays; this energy is such that a significant number of photons can escape from the body without being dispersed or attenuated, these are detected by an external system sensitive to position; this external system is the GC

Corresponding author: Jorge Luis Domínguez Martínez, bachelor, professor, research fields: nuclear instrumentation and medical physics. (gamma camera), whose basic function is to provide an image of the activity distribution of the radiopharmaceutical administered to the patient. Commonly, images produced by GCs are called images produced by radionuclides [2, 4]. There are two types of imaging methods in NM: by single photon emission (planar GC and SPECT) and by positron emission (PET) [3]. Up to 2012, more than 30 million images had been produced in NM, with more than 20,000 GC (between planar and SPECT) and more than 3,000 PET [1]; Today, Cuba has NM services in 10 provinces of the country (see Fig. 1).

Sometimes the service provided by the GC in the departments of NM, is unusable due to damage to them, in most cases due to the associated electronics and not to the physical components of detection. For this reason, it has been decided to develop an electronic system that allows the operation and optimization of them beginning with the design of the preamplifier for the signal of each PMT. It is proposed that the design and simulation of a preamplifier card for the signals generated by the GC's PMT contribute to the recovery and optimization of disused gamma cameras for their subsequent clinical use. 


\section{Materials and Methods}

A planar gamma camera (GC) or a SPECT type, basically consists of a radiation detector head (see Fig. 2) constituted by, a collimator that allows only the passage of radiation emitted by the radioisotope to be evaluated, a crystal of scintillation with a certain thickness and scintillating material, which is able to detect photons and emit light through the light guide, which serves as an optical coupling to improve uniformity, then they are transformed into an electrical impulse by PMT (photomultiplier tubes), to later be amplified and processed in terms of its spatial location, by means of an electronic system (preamplifier, pulse arithmetic, pulse height analyzer (SCA), analog-digital converter (ADC)) connected to a computer (PC) that allows to see and process the functional images (plane distribution map of the radiation in front of the detector) of the organ studied [4].

According to the type of electronics associated with the $\mathrm{GC}$ for the processing of the signals obtained from the PMT, this can be classified into: Analog, Hybrid (Analog-Digital) and Digital.

The detector is composed of a scintillation crystal of Sodium Iodide activated with Thallium (NaI (Tl))

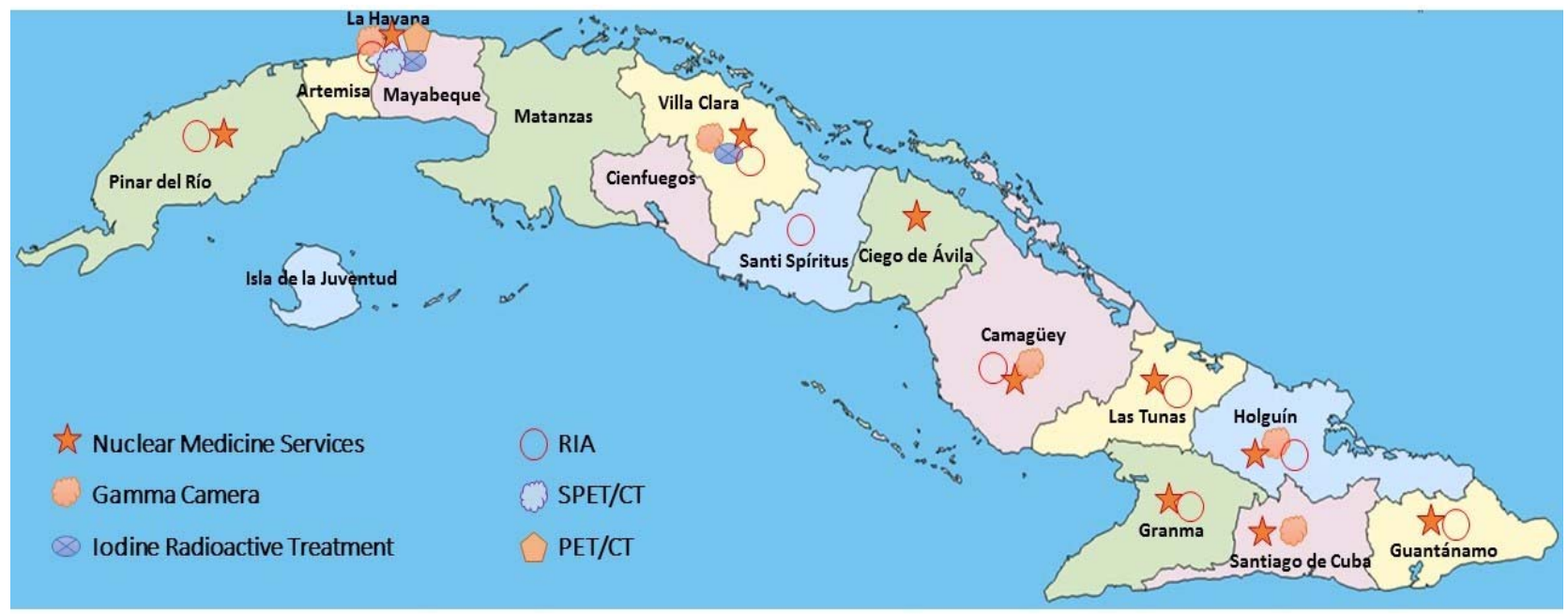

Fig. 1 Distribution of nuclear medicine services and equipment in Cuba.

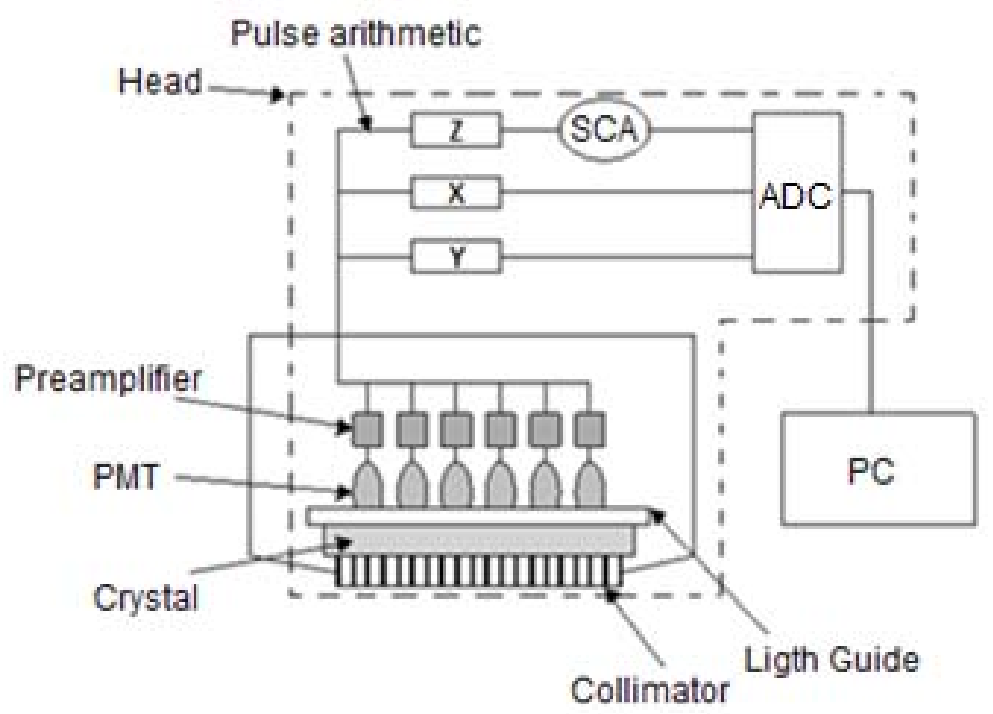

Fig. 2 Basic outline of a hybrid gamma camera. 
ideal for the range of energy $80-300 \mathrm{KeV}$, of variable thickness and size, coupled to a set of PMT by an optical coupling gel, which allows uniformity in the light emitted from the crystal towards the PMTs [1]. For the external detection it is necessary to select the radiation that comes from a small area or volume, for this reason the photons originated outside the area of interest are discarded, this is done by means of collimation, ensuring that only the detector reaches the gamma rays that coming at a specific angle with respect to the detector crystal. The pulse of light coming out of the glass is transmitted through the light guide (see Fig. 2), which allows it to only go in a direction parallel to the PMT $(\sim 50 \mathrm{~mm}$ in diameter by $\sim 150 \mathrm{~mm}$ in length) without deviate [2]. These tubes have the function of converting light signals into electric amplitude proportional to the incident light; these signals are used to determine the position and energy of gamma rays. In the case of digital cameras, the light guide is usually removed $[1,2]$. The arrangement of PMT varies depending on the type of head according to the age of the camera, typically from 60 to 90 PMT present the modern GC [4] (in Cuba you can find equipment from 37 to 91 PMT of 2 or 3 inches of diameter) and the size of the visual field of it; you get better resolution with more small PMTs than with less large tubes. The electrical current generated by the PMT is very low voltage (small amplitude), it needs to be amplified several volts before they can be analyzed or processed. In the case of NaI (Tl) with PMT present an output signal of 100 $\mathrm{mV}$ to $1 \mathrm{~V}$ with duration of $0.23 \mu \mathrm{s}$. A typical preamplifier in NM presents a time constant between 20 and $200 \mu \mathrm{s}$; long time constants are ideal in systems that require optimal energy resolution [1]. The most important properties of a preamplifier are gain, bandwidth, linearity, dynamic range, slew rate, rise time, ringing, saturation, stability and noise [2]. The gain is defined by the ratio $P_{o}$ (power/output voltage) and $P_{i}$ (power/input voltage) usually given in decibels:

$$
G[d b]=10 \log \left(\frac{P_{o}}{P_{i}}\right)
$$

Modern GCs are completely digital, in essence the output signal of the PMT is directly (without using preamplifier) digitized by an ADC (analog-digital converter); the $\mathrm{XY}$ position calculation and the pulse height analyzer is performed by software based on the digitized signal of the PMT [4]. In our case, a programmable amplification stage using a DAC (digital-analog converter) will be used in the preamplifier to adjust the gain of the PMTs by software. To choose the DAC that will be used, its setting time, accuracy, linearity, offset error, monotonicity and resolution must be taken into account [5], the latter depends on the external reference voltage $\left(V_{\text {ref }}\right)$ and the number of bits $(N)$.

$$
\text { resolucón }=\frac{V_{r e f}}{2^{N}}
$$

To test the operation of the DAC, the static accuracy test and the staircase test will be used [5].

All the GCs according to their stage of evolution, have a console (PC) or operating device (see Fig. 2), connected to this unit are the devices for control and processing of images and data, which allow the development of the activity diagnostic. Currently in Cuba all the services that have GC have computerized units that allow acquiring, processing and archiving the studies carried out, since the oldest equipment has been updated with the Imagamma system (consisting of an electronic card and software).

\section{Results and Discussion}

The designed preamplifier (see Fig. 3) consists of five amplifying stages; in the case of the programmable Amp, a DAC is responsible for varying the gain in a programmed manner and, together with the rest of the blocks in the circuit, forming the signal for subsequent digitization.

In the Amp $\times 100$ (see Fig. 4) we used an inverter amplifier with simple input operation that works as a constant gain multiplier (AD829) and also a current 


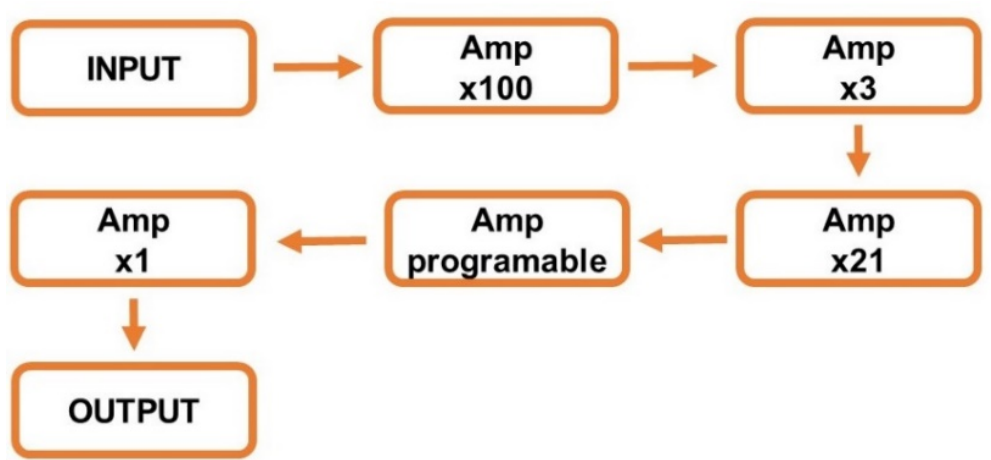

Fig. 3 Block diagram of the preamplifier.

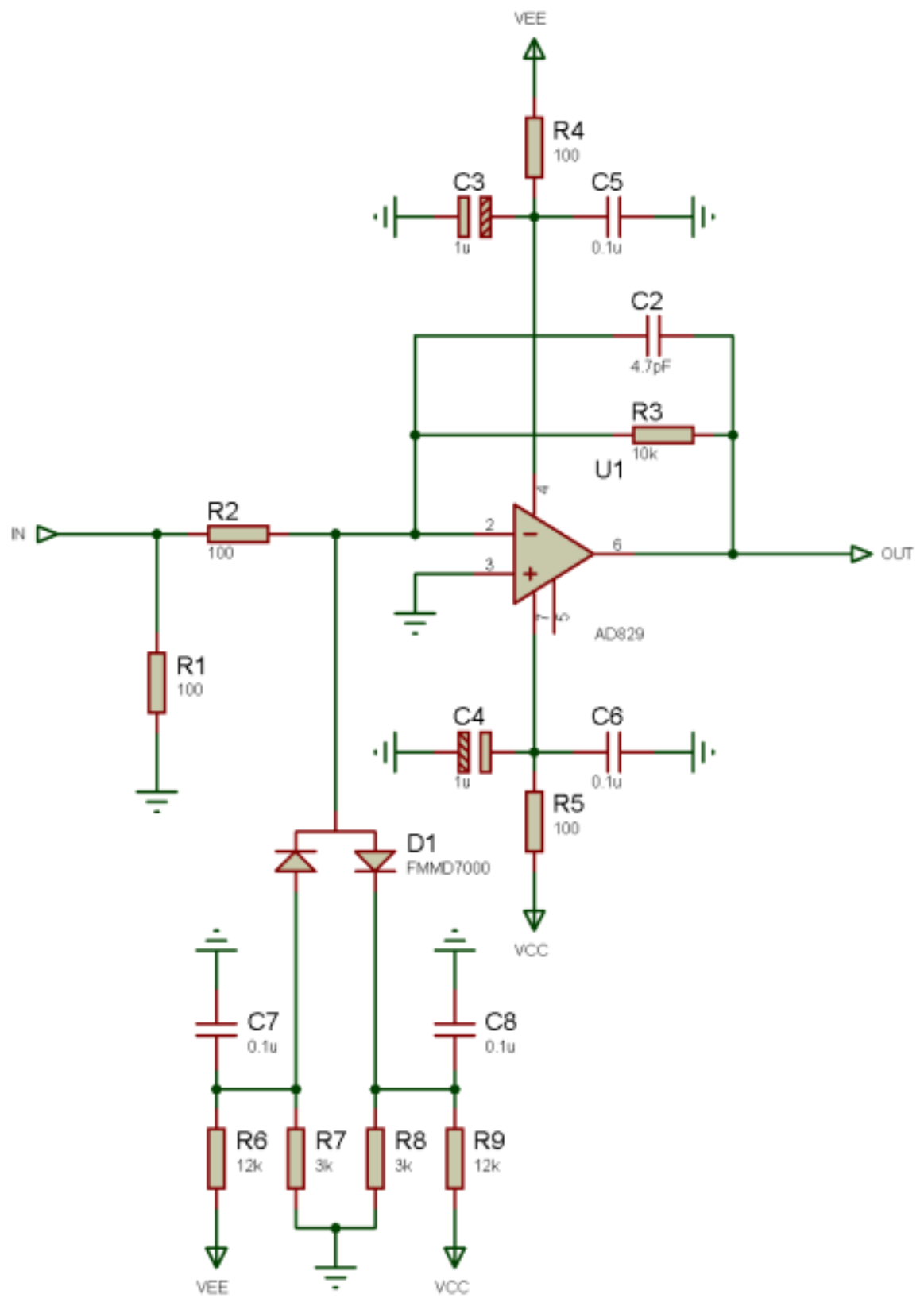

Fig. 4 Diagram of the stage Amp $\times 100$. 
limiter from the pair of silicon diodes in opposition (FMMD7000) for the protection of the input against overvoltage [6].

The AD829 is chosen for its speed (120 MHz), slew rate $(230 \mathrm{~V} / \mu \mathrm{s})$ and its low settling time (90 ns) [7]. Many Amp-Ops increase the speed as the slew rate decreases, but in the case of the AD829 it gives a good precision in the amplification of the current pulses for the first stage of the circuit and stability in the baseline for high rates of counts. At the input of this circuit, a resistance of $100 \Omega$ is placed. It together with the $10 \mathrm{k} \Omega$ feedback resistance produces a gain of 100 and a phase inversion of $180^{\circ}$ for this stage of the circuit. The gain can be affected by the temperature or other factors of the circuit, SO or SMD (Surface Mount Device) resistance will be used very thin with low dissipation of power of $1 / 8 \mathrm{~W}$, with a high precision and high stability of the RT series of type 0805 [8] and SO capacitors with high precision and high resistance to humidity $[9,10]$. It was considered not to perform a filtering of the PMT signals, but only a small period integration ( $47 \mathrm{~ns}$ ) and sufficient linear amplification, to perform the digitization. This method is widely used since it requires few components and is more suitable due to the large number of electronic channels these systems have.

In the Amp $\times 3$ (see Fig. 3) an inverter amplifier with simple input operation that works as a constant gain multiplier (LT1365) was used. At the input of this circuit, a resistance of $1 \mathrm{k} \Omega$ is placed. It together with the $3 \mathrm{k} \Omega$ feedback resistance produces a gain of 3 and a phase inversion of $180^{\circ}$ for this stage of the circuit. The LT1365 is chosen because it brings four operational amplifiers in the same package to have a high density and integration that would allow circuits to be made in the minimum space; in addition, it is chosen for its speed $(70 \mathrm{MHz})$, high slew rate $(1,000$ $\mathrm{V} / \mu \mathrm{s})$ and for maintaining stability in the baseline for high count rates [11].

In Amp ×21 (see Fig. 3), a non-inverting amplifier with simple input operation was used that functions as a constant gain multiplier (LT1365). A resistance of 1 $\mathrm{k} \Omega$ can be appreciated together with the feedback resistance $20 \mathrm{k} \Omega$ produce a gain of 21 , maintaining the phase for this stage of the circuit.

The gain for the amplifier stages (see Fig. 5) is kept constant for a wide range of frequencies that could be simulated, which allows the use of the preamplifier with frequencies up to $1 \mathrm{MHz}$, in this way to be able to modernize the GCs that work with $200 \mathrm{kHz}$ and $500 \mathrm{kHz}$ frequency regimes such as Siemens Orbiter and Siemens e.Cam respectively $[12,13]$. The overall gain for the first three stages Amp $\times 100, A m p \times 3$ and Amp $\times 21$ is 1999 .

In the Amp $\times 1$ (see Fig. 3) an amplifier (LT1365) was used, which has as input the Amp programmable output signal with unity gain. This circuit serves as a coupling to the $\mathrm{ADC}$ for the subsequent digitization of the pulses.

For the design of this Amp programmable stage, a DAC with sufficient resolution and a fast response with $\mathrm{N}$ greater than or equal to 12-Bit are used. Several models of Maxim and Analog Device were simulated only fulfilling the necessary requirements: the MAX543, MAX5541 and AD5410. Of these, the MAX5541 (see Fig. 6) was chosen for being the most economical and resolutive (16 bits), on the other hand, its settling time $(1 \mu \mathrm{s})[14]$ is not a problem since the pulses of the PMTs of the GC have a duration of 1.2 $\mu \mathrm{s}$.

The advantage is that the DAC is a multiplier because can use a variable analog signal $\left(V_{\text {ref }}\right)$ instead of a fixed reference voltage; this allows to use the DAC in programmable amplifier/attenuator, automatic calibrations, gain adjustments, among others. [5, 14]. The serial data transfer signals with the DAC are determined by the clock signal (CLK), serial data (SRI) and synchronization (LOAD). The output voltage for this stage is given by:

$$
V_{\text {OUTPUT }}=V_{R E F} *\left(\frac{S R I_{d}}{2^{N}}\right)
$$




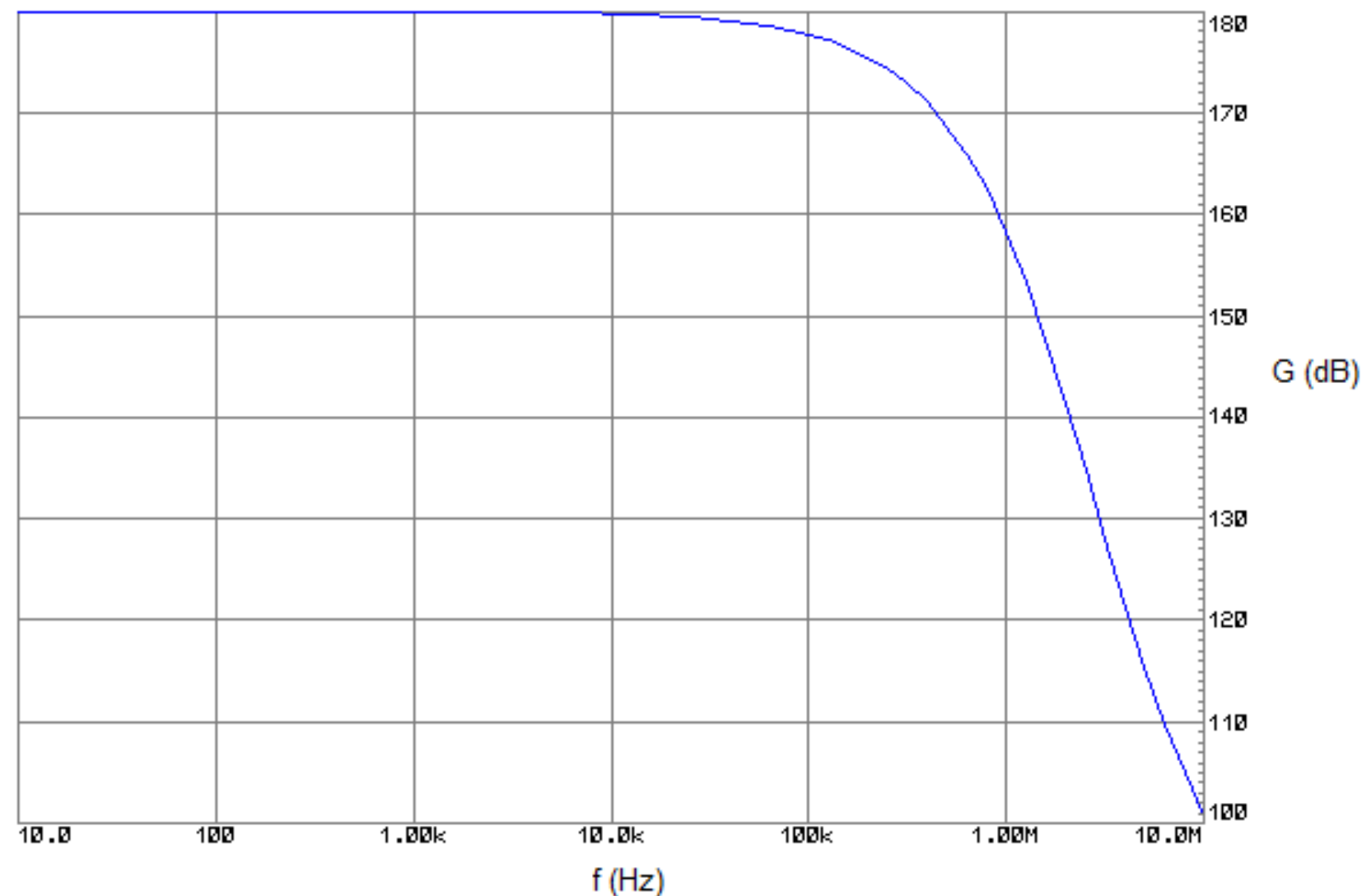

Fig. 5 Dependence on the gain of amplifying stages vs. frequency of the input signal.

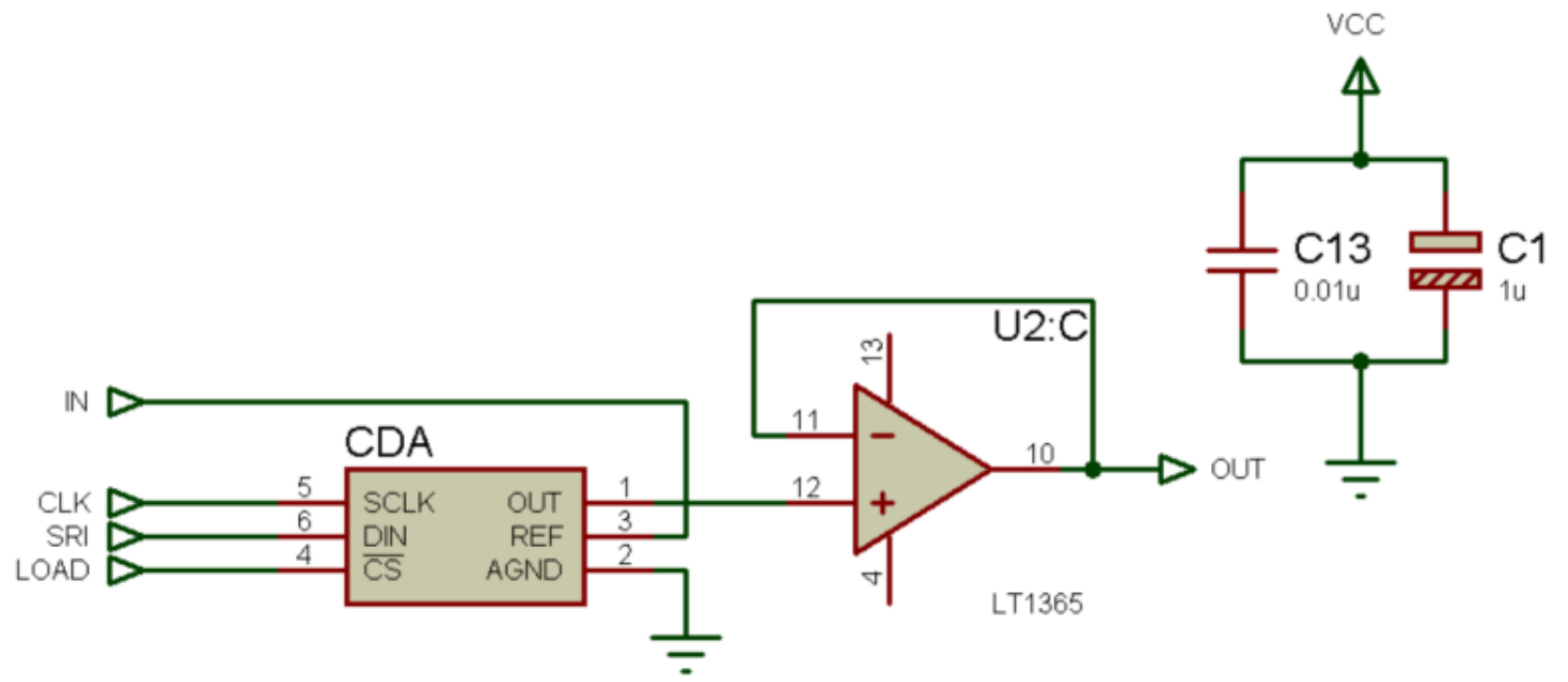

Fig. 6 Diagram of the stage amp programmable.

Where $N$ is the bit number and $S R I_{d}$ is the serial input expressed in decimal. In general, the preamplifier will consist of a DAC MAX5541, an AmpOp AD829 and an integrated circuit (IC) with four Amp-Op, the LT1365. In principle this preamplifier will be used in the GC that are in disuse, but that the detector head (only collimator, crystal and PMT) is in good physical condition. 
For the simulation of the designed circuit, Proteus 8 Professional (Release 8.0 SP0, build 15417) was used, from the company $\left.{ }^{(}\right)$Labcenter Electronics 1989-2012 with advanced simulation. With the ISIS module (Intelligent Schematic Input System) of Proteus, the schematic captures were made and with the ARES module (Advanced Routing and Editing Software) of Proteus, the PCB (printed circuit board) was designed. Proteus from the VSM (Virtual System Modeling) allows a simulation design in real time.

For the simulation of the preamplifier, the PMT signal was replaced by a pulse generator with the following characteristics: duration of $1.2 \mu \mathrm{s}$, amplitude of $10 \mu \mathrm{A}$ frequencies from $200 \mathrm{KHz}$ to 1 $\mathrm{MHz}$. The lower limit frequency of $200 \mathrm{KHz}$ is chosen because the GC Orbiter has that average processing frequency, on the other hand, $500 \mathrm{KHz}$ is chosen because it is the average processing frequency of the Siemens e.Cam SPECT; in addition, it is tested with faster frequencies such as $1 \mathrm{MHz}$ to facilitate the optimization and modernization of GCs that will use these cards. It is tested with several values of the SRI signal of the DAC for different frequencies $(200 \mathrm{kHz}$, $500 \mathrm{kHz}$ and $1 \mathrm{MHz}$ ) and amplitudes of the input signal $(10 \mu \mathrm{A}$ and $15 \mu \mathrm{A})$. A clock signal with a

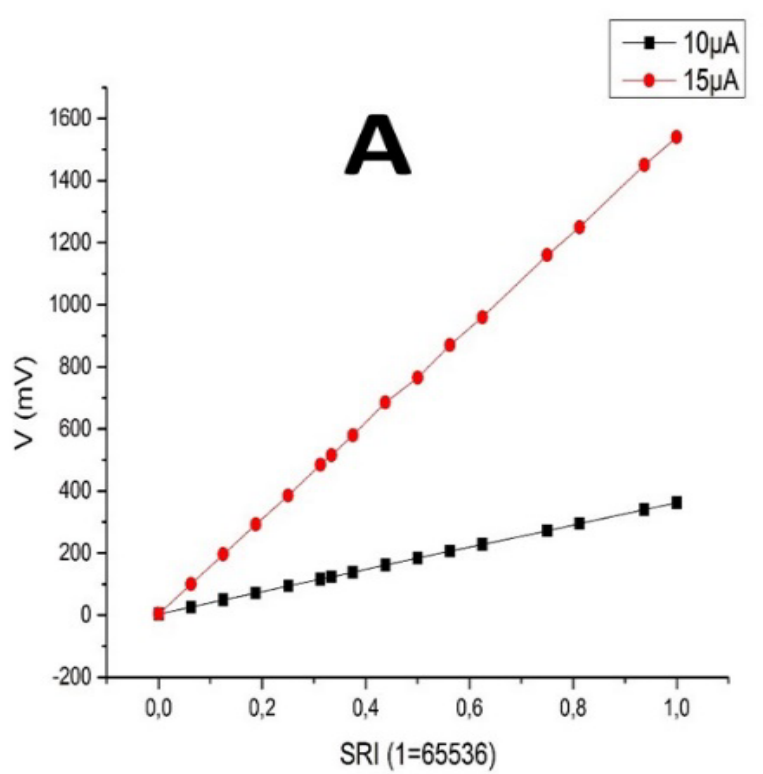

frequency of $22.5 \mathrm{~ns}$ was used, the signal LOAD and SRI with pulses of $45 \mathrm{~ns}$ both in high (1) or low (0).

The output of the preamplifier follows a linear behavior for $200 \mathrm{kHz}$ (see Fig. 7A); the linear adjustment of $10 \mu \mathrm{A}$ of amplitude for the input signal corresponds to the equation

$$
y=358.2727 x+4.04743
$$

with $\mathrm{R}^{2}=0,99998$ and an error of 0.24183 and 0.44541 for the intercept and the slope respectively; for the $15 \mu \mathrm{A}$ linear adjustment (see Fig. 7A) the equation is given by

$$
\mathrm{y}=1,538.23421 \mathrm{x}+3.27846
$$

with $\mathrm{R}^{2}=0.99993$ and an error of 1.76364 and 3.24831 for the intercept and the slope respectively. The linear adjustment was made with OriginPro 9.0.0 (64-bit) SR2 Copyright 1991-2013 OriginLab Corporation in its version for Windows.

The output of the preamplifier with the increase of the frequency of the input signal is not affected (see Fig. 7B). This follows a linear behavior; a check of the monotonicity of the DAC can be observed for the staircase test, since there is a decrement in the voltage for a decrement in the SRI step by step. In the case of the static test for different frequencies, almost the same voltage value is obtained at the output of the

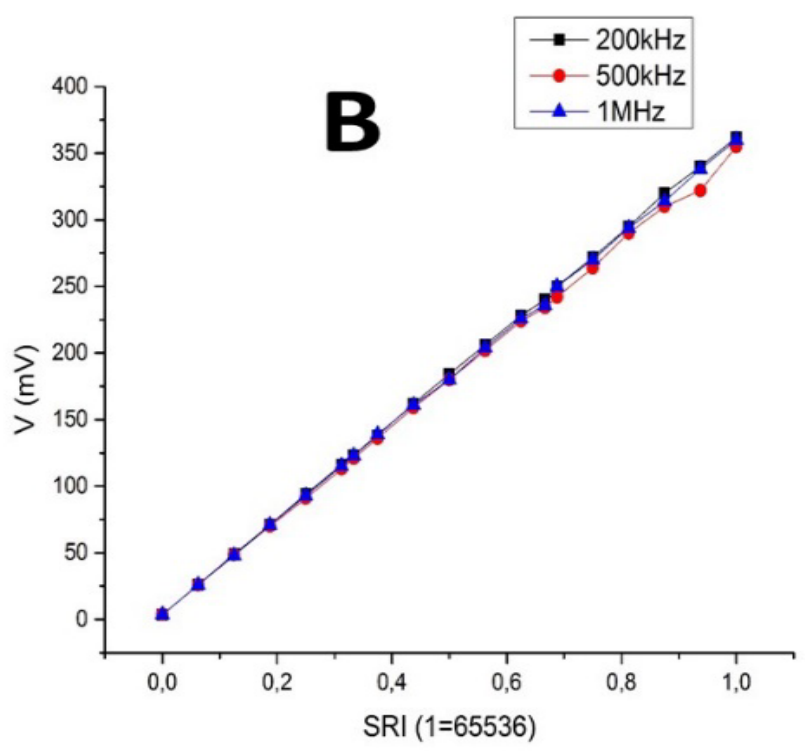

Fig. 7 Output voltage of the preamplifier vs. SRI of the DAC: (A) for $10 \mu \mathrm{A}$ and $15 \mu \mathrm{A}$ of amplitude of the input signal at $200 \mathrm{kHz}$, (B) for $10 \mu \mathrm{A}$ of amplitude for the input signal at $200 \mathrm{kHz}, 500 \mathrm{kHz}$ and $1 \mathrm{MHz}$. 


\section{PCB Layout}

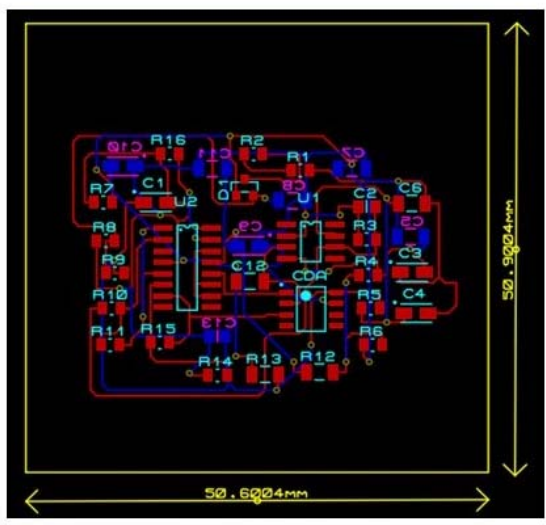

\section{Front}

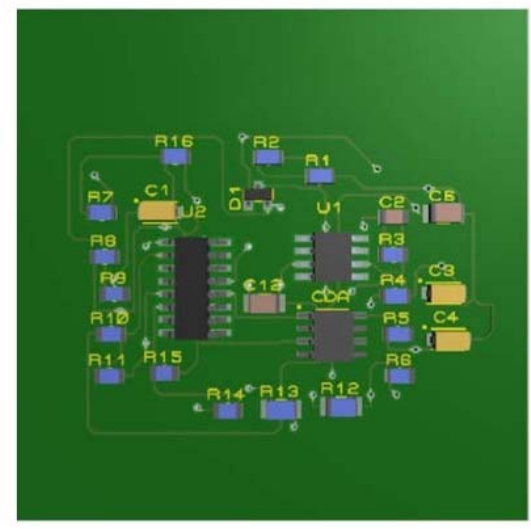

Back

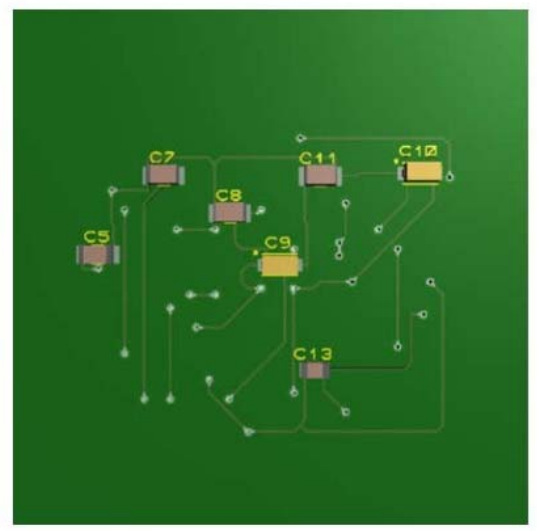

Fig. 8 Layout and 3D diagram of the printed circuit board with the components of the preamplifier.

preamplifier for fixed SRI inputs. For $500 \mathrm{kHz}$ the linear adjustment corresponds to the equation

$$
\mathrm{y}=346.81768 \mathrm{x}+5.06484
$$

with $\mathrm{R}^{2}=0.99937$ and an error of 1.18911 and 2.04896 for the intercept and the slope respectively; for $1 \mathrm{MHz}$ the equation is given by

$$
y=355.32884 x+4.004
$$

with $\mathrm{R}^{2}=0.9998$ and an error of 0.69156 and 1.19163 for the intercept and the slope respectively.

The default options of the Proteus routing were used to obtain the printed circuit of the preamplifier. The size was chosen from the smallest width of the GC's PMT that exist in Cuba, which varies from 2 to 3 inches wide with round and hexagonal shapes. A square shape was chosen for 1 PMT to facilitate its assembly. The ARES and 3D Visualizer function was used to show the circuit according to its physical components (see Fig. 8).

\section{Conclusions}

A preamplifier card for the photomultiplier tubes of a Gamma Camera was designed and simulated. The list of components necessary for the construction of the preamplifiers was generated. During the simulations, the preamplifier cards maintained a linear behavior. The printed circuit board for a preamplifier was designed for future construction and configuration. These cards could replace all the preamps used in the current GC's PMTs. This allows automatic adjustment of the gain by software and not manual as in some current gamma cameras not modernized.

\section{References}

[1] SIMON R. CHERRY, JAMES A. SORENSON and MICHAEL E. PHELPS. 2012. Physics in Nuclear Medicine. Philadelphia: Elsevier Saunders, 1-6, 107-118, 195-208.

[2] BAILEY, D. L., HUMM, J. L., TODD-POKROPEK, A., and VAN ASWEGEN, A. 2014. "Nuclear Medicine Physics: A Handbook for Teachers and Students." IAEA, Vienna, 205-12, 222-6, 312-53, 392-7.

[3] IAEA. 2006. "Nuclear Medicine Resources Manual." IAEA, Vienna, 107-31.

[4] BROWN, B. H., SMALLWOOD, R. H., BARBER, D. C., LAWFORD, P. V., et al. 1999. "Medical Physics and Biomedical Engineering." Taylor \& Francis Group, LLC, 369-78.

[5] RONALD, J. TOCCI, NEAL, S. WIDMER and GREGORY L. MOSS. 2011. Digital Systems. Principles and Applications. New Jersey: Pearson Prentice Hall, 733-9.

[6] FMMD7000. 1995. SOT23 Silicon Planar High Speed Switching Diode Pair (Series). Zetex Semiconductors, England.

[7] AD829. 2000. High-Speed, Low Noise Video Op Amp. Datasheet. Analog Devices Inc., USA.

[8] YAGEO. 2017. Thin Film Chip Resistor. High Precision-High Stability. Datasheet. Yageo Phicomp, USA.

[9] PANASONIC. 2017. Products Catalogs. Plastic Film Capacitor. Datasheet. Panasonic, USA.

[10] T498. 2017. Solid Tantalum Chip Capacitors-High Temperature. Datasheet. Kemet Charged, USA. 
[11] LT1364/LT1365. 1994. Dual and Quad $70 \mathrm{MHz}, 1,000$ $\mathrm{V} / \mu \mathrm{s}$ Op Amps. Datasheet. Linear Technology Corporation, USA.

[12] Wolfgang Rebhan. 2000. CamControl V2.4. Installation Manual. Siemens AG, USA.
[13] e.Cam. e.cam Signatures Series. All about Quality, Speed and Comfort. Siemens Medical Solutions, USA. 2006.

[14] MAX5541. 1999. Low-Cost, +5V, Serial-Input, Voltage-Output, 16-Bit DAC. Maxim Integrated Products, USA. 